\title{
The effect of detraining on balance and risk of falls in older adults
}

\begin{abstract}
Objective: To evaluate the detraining of older adult women who exercise in the aquatic environment.

Method: A quantitative, retrospective study whose intervention was classified as almost experimental without control group, and the sample was composed of elderly women who exercise in a group in the aquatic environment. The evaluation of the sample counted with anamnesis and the tools Berg Balance Scale (BERG) and Timed Up and Go test (TUG). Two evaluations were carried out at different times, with the first evaluation at the beginning of the detraining period and the second evaluation, three months later, immediately before the participants returned to the exercise group. For statistical analysis the Shapiro-Wilk and Wilcoxon tests were applied.
\end{abstract}

Results: The study had 17 elderly women, mean age of $69.23 \pm 8.09$ years, residents of Santa Maria town, RS. The balance variable remained the same between the two evaluations $(\mathrm{p}=0,655)$, that is, there was no equilibrium shortage related to detraining. The risk of falls showed a significant improvement in scores when compared to the 1st evaluation with the 2nd evaluation $(\mathrm{p}=0,01)$

Conclusion: The results of the study suggest that the dual tasks exercises, performed in the aquatic environment continue to positively influence the variables after three months of detraining.

Keywords: exercise, postural balance, older adults, double task
Volume 4 Issue 5 - 2019

\author{
Pietro Bataglin,' Clandio Timm Marques, ${ }^{2}$ \\ Carla Mirelle Giotto Mai, ${ }^{3}$ Lilian Oliveira \\ de Oliveira, ${ }^{4}$ João Rafael Sauzem Machado, ${ }^{5}$ \\ Jaqueline de Fátima Biazus, ${ }^{6}$ Alethéia Peters \\ Bajotto $^{7}$ \\ 'Academic of the Physiotherapy course, Franciscan University, \\ Brazil \\ ${ }^{2} \mathrm{PhD}$ in Biostatistics and Bioinformatics, Professor of \\ Mathematics at the Franciscan University, Brazil \\ ${ }^{3}$ Master in Toxicological Biochemistry, Federal University of \\ Santa Maria, Canada \\ ${ }^{4} \mathrm{PhD}$ in Science Education, Chemistry of Life and Health, \\ Professor of the Physiotherapy course at the Franciscan \\ University, Brazil \\ ${ }^{5}$ Master of Human Communication Disorders, Professor of the \\ Physiotherapy course at the Franciscan University, Brazil \\ ${ }^{6}$ Master in Public Health, Professor of the Physiotherapy course, \\ Franciscan University, Brazil \\ ${ }^{7} \mathrm{PhD}$ Medicine, Medical Sciences URGS, Professor of the \\ Physiotherapy course, Franciscan University, Brazil
}

Correspondence: Jaqueline de Fatima Biazus, Master in Public Health, UNISUL, Professor of the Physiotherapy course, Franciscan University - Santa Maria (RS), Brazil, Tel +5555996199919, Email jaquebiazus@hotmail.com

Received:September 12, 2019 | Published: October 01, 2019

\section{Introduction}

Currently, it is estimated that over 900 milion people are over 60 and it is projected that by $205021.5 \%$ of the world's population will be elderly. In Brazil, there was a 30.3 year increase in life the expectation and estimate for 2050 is 80 years old, expected to be the sixth country in the ranking, with more elderly people than normal people. $^{1,2}$

According to World Health Organization, ${ }^{3}$ a minimum of 150 minutes of physical activity should be performed weekly for positive effects on the cardiovascular system and skeletal muscle. Exercise is considered the most effective way to prevent and treat major conditions and limitations, including cardiovascular disease and cognitive deficiencies such as Alzheimer's disease. ${ }^{4,5}$

Individual intrinsic changes such as decreased ability to maintain postural balance (influenced by factors such as cognitive impairment, unsteady gait, and muscle weakness) associated with extrinsic factors increase the risk of falling in the elderly population. These falls associated with increased reaction time and pathologies such as osteoporosis, which have its highest incidence in this age group, lead to higher rates of health spending, long-term (or permanent) functional disability and mortality due to these condition falls are generally influenced by balance deficits. ${ }^{6,7}$
The relationship between balance and risk of falls includes intrinsic and extrinsic factors. concomitantly with adaptations of extrinsic factors, one of the main resources for reducing the risk of falls is physical activity performed regularly to reduce intrinsic influences, such as muscle weakness. ${ }^{8,9}$

Thus, specialists focus on the variables of time and intensity of training necessary to gain benefits due to the practice of physical exercises. However, there is still little emphasis on the effects of the detraining period, that is the time when the individual interrupts the practice of physical exercises. ${ }^{10}$

This research study has been carried out to evaluate the detraining in the age group of the elderly, and its effects on the variables of balance and risk of falls in the elderly.

\section{Methodology}

A quantitative, retrospective study, whose intervention was classified as quasi-experimental without control group, where measurements were made before and periodically after the was carried out. The population consisted of elderly women living in the city of Santa Maria, RS. The sample was of the accidental, non-probabilistic type. 
Inclusion factors were: 60 years or older; carriers of chronic non-communicable disease (CNCD); achieve at least 23 points in the Mini Mental State Exam (MEEM); perform moderate physical activity, attested by a doctor (last year); able to travel to the Teaching and Research Laboratory (LEP), from the Franciscan University. Exclusion criteria included elderly women who: a) performed any other physical exercises concomitant with the activities proposed by this study ; presented decompensated cardiopulmonary condition; used walking aids; were unable to complete all stages of the informed consent process for participation in the research.

Participants performed the aquatic exercise in the heated pool of the Franciscan University Teaching and Research Laboratory (LEPUFN) of Santa Maria, beginning in September 2017. The physical therapy intervention took place twice a week, lasting 45 minutes each session, in a heated pool. Participants were assessed using an assessment form, the BERG balance scale and the TUG test that assesses the risk of falls.

The data analyzed refer to the participants assessment in September 2017, revaluation in November 2017, 3-month interval (detraining) and revaluation performed in February 2018.

The activities performed in the coexistence group recommended the use of the double task. Examples of activities that were used with the elderly group are: in group, while doing squats, counting prospectively and retroactively until certain numbering; Board-guided dance activities with different meanings.

The group session followed a chronological order:

$$
\begin{aligned}
& \text { i. Heating; } \\
& \text { ii. Dual task activities; } \\
& \text { iii. General strengthening; } \\
& \text { iv. Stretching; }
\end{aligned}
$$

To assess whether there was detraining in the group, we used the BERG scale, which consists of the risk of falls, the TUG test. The results obtained in November 2017 and then in February 2018 were analyzed. To test the normality of the variables, the Shapiro-Wilk test was used. As normality was compromised, the nonparametric Wilcoxon test was used to compare the two time-points. Differences were considered significant when the results presented $p$-value $<0,05$.

The activities began after the study was approved by the institution's Research Ethics Committee (CEP) under number 2.196.717.

\section{Results}

The sample consisted of 17 elderly women, with a mean age of $69,23 \pm 8,09$ years, most of them were married $(70,59 \%)$, White $(88,23 \%)$ and residents of Santa Maria, RS.

The overall average of the balance assessment performed using the BERG scale in November 2017 was 55,24 $\pm 2,25$ and the average in March 2018 was 55,06 $\pm 2,72$. As shown in Figure 1, it appears that of the 17 participants in the study, 15 maintained the same balancerelated score, for one participant the score increased and for another elderly, the score decreased. There is no statistical evidence to affirm that there was a detraining in the evaluated group, as measured by the BERG scale (Wilcoxon $Z=-0.477$; p-value $=0.655$ ).

As shown in Figure 2, of the 17 patients in the study, 13 (76.4\%) presented a reduction in TUG (reduction in risk of falls) from the first to the second evaluation, while four $(23.6 \%)$ increased after three months of detraining. In the analysis of the two time-points analyzed, we found that there was a significant difference between assessment $1(11.17 \pm 3,29)$ and assessment $2(9,61 \pm 1,84)$ (Wilcoxon $Z=-2,58$; p-value $=0,01)$.

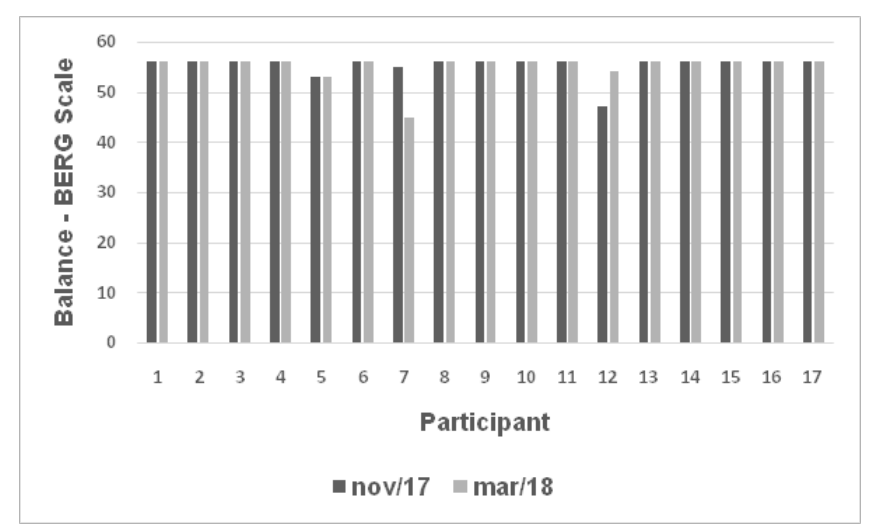

Figure I Values of evaluations performed with the BERG scale in Nov/I7 and Mar/l 8 .

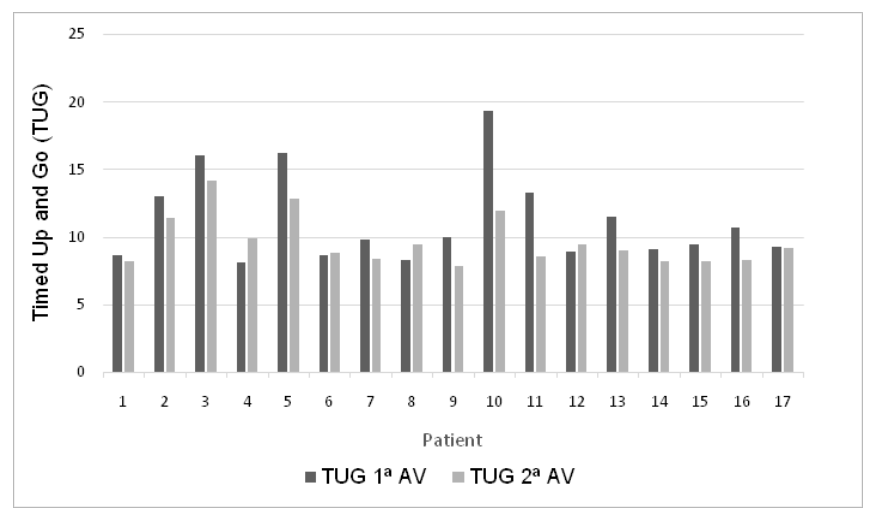

Figure 2 Values of evaluations performed with the TUG scale in Nov/ I7 and Mar/l8.

\section{Discussion}

Regular exercise consisting of aerobic exercise associated with resistance exercise can achieve results of up to 30 years improvement in the physical performance of individuals, active 80 -years-olds can perform the same exercise as sedentary people in the 50-year age group. ${ }^{11}$

Detraining can be characterized as the period in which the stimuli provided by physical exercises are interrupted, that is, the period in which the subject ceases to perform the training. ${ }^{12}$ The repercussions of this recess period are varied and are influenced by the physiology of the individual and the method used in the application of training, such as its intensity, durability and types of exercises performed. ${ }^{13}$ Balance is a determining variable for healthy aging. In situations of balance deficit the chances of secondary consequences such as reduced mobility and falls increase, generating a negative predictor for the health of the elderly. ${ }^{14,15}$

In this study, which evaluated the three months detraining of a group of elderly women who performed physical exercises in the aquatic environment, the balance variable showed no loss of performance in the test. The result showed that there was no significant difference between the two evaluation time-points. 
The dual-task exercises, which were used as the basis for the training of the elderly participants of the research protocol, seem to positively impact on cognitive training and, ultimately, the improvement or maintenance of cognition, improving balance and consequently, preventing falls. ${ }^{16}$

We can consider that the effects of physical exercise may persist even after a certain period of withdrawal from training, as in the research by Teleniu et al. ${ }^{17}$ who applied for three months (twice a week), an intervention composed of strengthening and balance exercises in 87 elderly who were part of the intervention group and were affected by some degree of neurological dysfunction. The results, using the BERG scale for the evaluation, showed that the gains regarding balance were maintained after three months of detraining, which is in accordance with the findings of the present study.

The other variable analyzed was the risk of falls, when related to the elderly, falling is interpreted as a loss of balance due to motor and/ or cognitive issues. ${ }^{18}$

In the detraining period evaluated in this research protocol, the result shows that this time without activity did not worsen the variable in question. This study presented statistical significance that proved the improvement in performance related to detraining period, that is, the means related to the TUG test show that the risk of falls decreased during the detraining period.

The result found in the present study corroborates the research conducted by Lacroix et al. ${ }^{19}$ who applied balance and strength training for three months and, after a period of three months from the end of the activities, it was still possible to find the beneficial effects on the risk of falling participants who as in the present research, were evaluated by the TUG test.

This result can be considered encouraging, as short periods of detraining may not decrease the gains resulting from months of guided training.

This important variable - detraining - is investigated in patients of different age groups age groups and physical conditions, as well as in each study, varying motor valences are analyzed as in the research carried out by Donti and his collaborators, ${ }^{20}$ who evaluated the flexibility of hip extensors in 57 young gymnasts applying stretching protocol 3 times a week for 15 weeks. When reevaluating the athletes after two weeks of detraining, it was observed that the gains from the intervention were maintained. Seco et al. ${ }^{21}$ developed combined strength, flexibility and balance training in 227 elderly (54 men and 173 women) over nine months and when reassessing after three months of detraining, verified the continuity of gains in the balance and flexibility variables.

As there is no consensus in the literature about detraining period for each type of training, it is possible to find a vast difference in the methodologies applied in research on the subject. Carvalho \&Marques, ${ }^{22}$ applying an eight-month training, with a sample of 32 elderly women, composed of aerobic exercises, strength, balance and flexibility, evaluated the results after three months of detraining and found the loss of all gains from the activity period. In this sense, Eggenberger et al..$^{23}$ who performed six months of intervention and 12 months of detraining, performing a double-task training in 47 elderly over 70 years, showed that although there was a decrease in the gains of some variables of gait (like speed), ambulation performance was still higher than that found in the pre-intervention period. Both studies aimed to improve physical and cognitive performance in elderly, however, their research application methodologies were extremely different.

\section{Conclusion}

Although the balance of the elderly participants in the sociotherapeutic group was maintained after a detraining period of three months, the risk of falls decreased during this same evaluated period. It is possible to state that the protocols developed by the authors, which mainly contemplated double task exercises performed in the aquatic environment, seem to continue to have a positive influence on both variables analyzed in this study.

\section{Funding}

None

\section{Acknowledgments}

None.

\section{Conflicts of interest}

Authors declare that there is no conflict of interest.

\section{References}

1. Brasil 2050: Challenges of an aging nation. chamber of deputies. Brazil; 2017.

2. World health organization (WHO). World aging and health report. 2015.

3. World health organization (WHO). Global recommendations on physical activity for health; 2010.

4. Beckett MW, Ardern CI, Rotondi MA. A meta-analysis of prospective studies on the role of physical activity and the prevention of Alzheimer's disease in older adults. BMC Geriatrics. 2015;15(9):1-7.

5. Sofi F, Valecchi D, Bacci D, et al. Physical activity and risk of cognitive decline: a meta-analysis of prospective studies. Journal of Internal Medicine. 2011;269(1):107-117.

6. Blomkvist AW, Eika F, Rahbek MT, et al. Reference data on reaction time and aging using the nintendo wii balance board: a cross-selectional study of 354 subjects from 20 to 99 years of age. PLOS One. 2017;12(12):1-13.

7. Wang J, Chen Z, Song Y. Falls in aged people of the Chinese mainland: epidemiology, risk factors and clinical strategies. Ageing Research Reviews. 2010;9(1):13-17.

8. Campbell AJ, Robertson MC. Rethinking individual and Community fall prevention strategies: a meta-regression comparing single and multifactorial interventions. Age and Ageing. 2007;36(6):656-662.

9. Rubenstein LZ. Falls in older people: epidemiology, risk factors and atrategies for prevention. Age and Ageing. 2006;35(2):37-41.

10. Esain I, Rodriguez-Larrad A, Bidaurrazaga-Letona I, Gil SM. Healthrelated quality of life, handgrip strength and falls during detraining in elderly habitual exercisers. Health and Quality of Life Outcomes. 2017;15(1):226

11. Landi F, Calvani R, Picca A, et al. Impact of habitual physical activity and type of exercise on physical performance across ages in community-living people. PLOS One. 2018;13(1).

12. Ivey FM, Tracy BL, Lemmer JT, et al. Effects of strength training and detraining on muscle quality: age and gender comparisons. $J$ Gerontol A Biol Sci Med Sci. 2000;55(3):152-157. 
13. Bae JY, Woo J, Kang S, et al. Effects of detraining and retraining on muscle energy-sensing network and meteorin-like levels in obese mice. Lipids in Health and Disease. 2018;17(1):97.

14. Nakagawa HB, Ferraresi JR, Prata MG, et al. Postural balance and functional Independence of elderly people according to gender and age: cross-sectional study. São Paulo Medical Journal. 2017;135(3):260-265.

15. Ribeiro AP, Souza ER, Atie S, et al. The influence of falls on the quality of life of the elderly. Revista Ciência \& Saúde Coletiva. 2008;13(4):12651273.

16. Fritz NE, Cheek FM, Nichols-Larsen DS. Motor-cognitive dual-task training in persons with neurologic disorders: a systematic review. Journal of Neurologic Physical Therapy. 2015;39(3):142-153.

17. Telenius EW, Engedal K, Bergland A. Long-term effects of a 12 weeks high-intensity functional exercise program on physical function and mental health in nursing home residents with dementia: a single blinded randomized controlled trial. BMC Geriatrics. 2015;15:158.

18. World health organization (WHO). WHO global report on falls prevention in old age. 2010.
19. Lacroix A, Kressig RW, Muehlbauer T, et al. Effects of a Supervised versus an Unsupervised combined balance and strength training program on balance and muscle power in healthy older adults: a randomized controlled trial. Gerontology. 2016;62(3):275-288.

20. Donti O, Papia K, Toubekis A, et al. Flexibility training in preadolescent female athletes: Acute and long-term effects of intermittent and continuous static stretching. Journal of Sports Sciences. 2017;36(13):1453-1460.

21. Seco, J, Abecia LC, Echevarría E, et al. A long-term physical activity training program increases strength and flexibility, and improves balance in older adults. Rehabilitation Nursing. 2013;38(1):37-47.

22. Carvalho MJ, Marques E, Mota J. Training and detraining effects on functional fitness after a multicomponent training in older women. Gerontology. 2009;55(1):41-48.

23. Eggenberger P, Theill N, Holenstein S, et al. Multicomponent physical exercise with simultaneous cognitive training to enhance dual-task walking o folder adults: a secondary analysis of a 6-month randomized controlled trial with 1-year follow-up. Clinical Interventions in Aging. 2015;10:17111732 . 